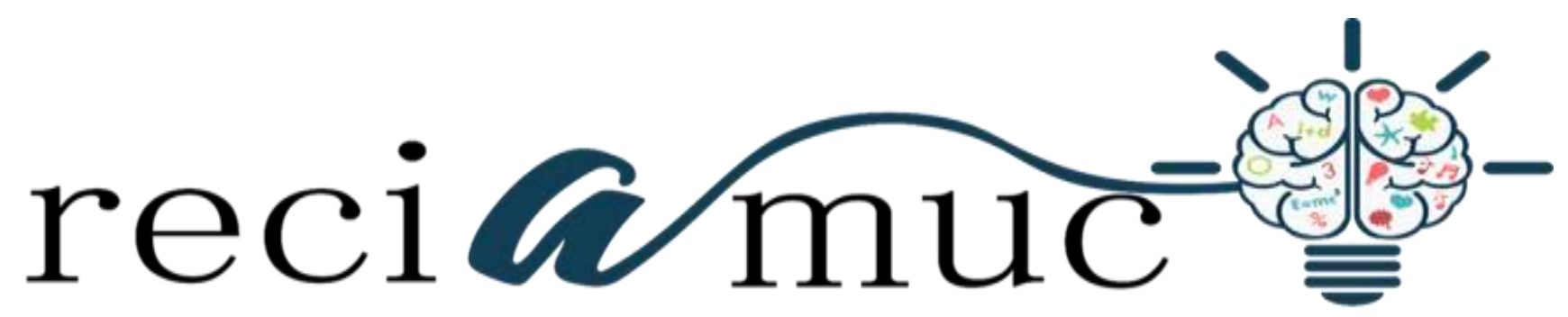

Revista cientifica de investigación actualización del mundo de las ciencias

José Fernando Calderón Ponce a ${ }^{\text {a }}$ César David Pibaque Tigua ${ }^{\text {b; }}$ Román Andrés Ortiz Burgasi ${ }^{c}$; Miguel Ángel Sánchez Rodríguez ${ }^{\mathrm{d}}$;

Luis Felipe Mendoza Alcívar ${ }^{\mathrm{e}}$; Maryury Beatriz Vinces Majojo ${ }^{\mathrm{f}}$

Procedimiento quirúrgico para pacientes sometidos a cirugía de trasplante renal Surgical procedure for patients undergoing kidney transplant surgery

Revista Científica de Investigación actualización del mundo de las Ciencias. Vol. 3 núm., 2, abril, ISSN: 2588-0748, 2018, pp. 996-1011

DOI: $10.26820 /$ reciamuc/3.(2).abril.2019.996-1011

URL: http://reciamuc.com/index.php/RECIAMUC/article/view/376 Código UNESCO: 3205 Medicina Interna

Tipo de Investigación: Artículo de Revisión

(C) RECIAMUC; Editorial Saberes del Conocimiento, 2019

Recibido: 15/01/2019

Aceptado: 07/02/2019

Publicado: 01/04/2019

Correspondencia: josecalderon1993@hotmail.com

a. Médico General; QUALIDENTAL S.A / MEDICALDENT; Guayaquil - Ecuador; josecalderon1993@hotmail.com

b. Médico general asistencial Hospital General Jipijapa.; Jipijapa - Ecuador; cesar_dav412@ hotmail.com

c. Médico Residente de cirugía plástica - Hospital General del Norte de Guayaquil Los Ceibos (IESS); Guayaquil - Ecuador; ballack1306@ hotmail.com

d. Médico General - QUALIDENTAL S.A / MEDICALDENT; Guayaquil - Ecuador; miguel-sanro @ hotmail.com

e. Médico Cirujano; Médico Rural de Puesto de Salud SAN MARCOS; Santo Domingo - Ecuador; lfmendoza1223@gmail.com

f. Médico de primer nivel de atención del distrito 23d02 Scs la modelo; Santo Domingo - Ecuador; marbevinces@gmail.com 


\section{Procedimiento quirúrgico para pacientes sometidos a cirugía de trasplante \\ renal}

Vol. 3, núm. 1., (2019)

José Fernando Calderón Ponce; César David Pibaque Tigua; Román Andrés Ortiz Burgasi; Miguel Ángel Sánchez Rodríguez; Luis Felipe Mendoza Alcívar; Maryury Beatriz Vinces

Majojo

\section{RESUMEN}

El trasplante renal consiste en la sustitución de los riñones nativos enfermos por un riñón sano, extraído de un donante vivo o muerto. En general todo paciente con insuficiencia renal terminal sin complicaciones o patologías graves asociadas que condicionen su supervivencia a corto plazo, con capacidad suficiente para entender y asumir el tratamiento, es candidato para recibir un trasplante renal. Como objetivos de esta valoración esta: la educación del paciente y su entorno familiar, detectar contraindicaciones absolutas y relativas, evaluar los factores de riesgo, evaluar el estado psicológico y la capacidad de adhesión al tratamiento y resolver las alteraciones encontradas antes del trasplante. Las técnicas más frecuentes de trasplante a partir de donador vivo: trasplante renal clásico o heterotópico: es la técnica quirúrgica más utilizada y sencilla, consiste en la colocación del riñón a trasplantar en la fosa ilíaca derecha por vía extra peritoneal. Trasplante renal en fosa ilíaca izquierda u ortotópico: era práctica frecuente colocar los riñones derechos en la fosa ilíaca izquierda con la finalidad de disponer la vía urinaria siempre para que resultara más fácil las reintervenciones (resolución de fístulas, estenosis etc.). Trasplante renal ortotópico izquierdo: describe una vía de abordaje extra peritoneal a los vasos esplénicos por Lumbotomía, utilizada inicialmente para la revascularización esplenorenal en la estenosis de la arteria renal, después para la derivación esplenorenal en la hipertensión portal y más tarde en el trasplante renal. La nefrectomía consiste en la extirpación quirúrgica de parte de un riñón (nefrectomía parcial), del riñón completo (nefrectomía simple) o del riñón, los ganglios linfáticos adyacentes y la glándula suprarrenal (nefrectomía radical). En una nefrectomía el cirujano corta los uréteres y los vasos sanguíneos y extrae el riñón. Puede hacerse con cirugía abierta o mediante laparoscopia. La ablación donante cadavérico se realiza en pacientes con muerte encefálica y aun con corazón batiente. La metodología usada es descriptiva, con un enfoque documental, es decir, revisar fuentes disponibles en la red, como google académico, con contenido oportuno y relevante desde el punto de vista científico que enriquezca el análisis del tema planteado en este artículo.

Palabras Claves: Transplante de Riñón; Donante Vivo; Donante Muerto; Laparoscopia; Ablación; Lumbotomía; Nefrectomía; Complicaciones. 


\title{
Procedimiento quirúrgico para pacientes sometidos a cirugía de trasplante renal
}

Vol. 3, núm. 1., (2019)

José Fernando Calderón Ponce; César David Pibaque Tigua; Román Andrés Ortiz Burgasi;

Miguel Ángel Sánchez Rodríguez; Luis Felipe Mendoza Alcívar; Maryury Beatriz Vinces

Majojo

\begin{abstract}
Renal transplantation involves the replacement of diseased native kidneys with a healthy kidney, extracted from a living or dead donor. In general, all patients with end-stage renal failure without complications or associated serious pathologies that condition their short-term survival, with sufficient capacity to understand and assume treatment, are candidates for receiving a kidney transplant. The objectives of this assessment are: the education of the patient and his family environment, to detect absolute and relative contraindications, to evaluate the risk factors, to evaluate the psychological state and the capacity of adhesion to the treatment and to solve the alterations found before the transplant. The most frequent transplant techniques from living donor: classic or heterotopic renal transplantation: it is the most used and simple surgical technique; it consists in the placement of the kidney to be transplanted in the right iliac fossa by extra peritoneal route. Renal transplantation in the left iliac or orthotopic fossa: it was common practice to place the right kidneys in the left iliac fossa in order to arrange the urinary tract always to make re interventions easier (resolution of fistulas, stenosis, etc.). Left orthotopic renal transplant: describes an extra peritoneal approach to splenic vessels by lumbotomy, initially used for splenorenal revascularization in renal artery stenosis, then for splenorenal bypass in portal hypertension and later in renal transplantation. Nephrectomy consists of the surgical removal of part of a kidney (partial nephrectomy), the entire kidney (simple nephrectomy) or the kidney, adjacent lymph nodes and the adrenal gland (radical nephrectomy). In a nephrectomy, the surgeon cuts the ureters and blood vessels and removes the kidney. It can be done with open surgery or by laparoscopy. The cadaveric donor ablation is performed in patients with brain death and even with a beating heart. The methodology used is descriptive, with a documentary approach, which is, reviewing sources available on the web, such as google scholar, with timely and scientifically relevant content that enriches the analysis of the topic raised in this article.
\end{abstract}

Key Words: Kidney transplant; Live Donor; Dead Donor; Laparoscopy; Ablation; Lumbotomy; Nephrectomy; Complications. 


\section{Procedimiento quirúrgico para pacientes sometidos a cirugía de trasplante \\ renal}

Vol. 3, núm. 1., (2019)

José Fernando Calderón Ponce; César David Pibaque Tigua; Román Andrés Ortiz Burgasi; Miguel Ángel Sánchez Rodríguez; Luis Felipe Mendoza Alcívar; Maryury Beatriz Vinces

Majojo

\section{Introducción.}

El transplante de riñón en palabras sencillas es colocar un riñón sano dentro del cuerpo de un paciente (diagnosticado previamente por un nefrólogo) para que realice el trabajo que sus propios riñones ya no pueden realizar. El transplante de riñón implica un alto grado de responsabilidad y compromiso para el enfermo, ya que deberá cuidarse de manera adicional: permanecer lo más saludable posible y tomar todos sus medicamentos diariamente.

Existen numerosos procedimientos quirúrgicos para realizarlo, los profesionales médicos le realizarán una serie de pruebas y radiografías para conocer su estado general de salud. Se analizará todo lo que pueda afectar cuán bien usted puede manejar el tratamiento. El trasplante de riñón puede hacerse a través de un donante vivo o uno muerto. Si tiene algún conocido que quisiera donarle un riñón, esa persona también necesitará realizarse una evaluación para averiguar si es compatible.

Plantear la posibilidad de realizar cirugía renal conservadora (tumorectomía, heminefrectomía) en aquellos casos seleccionados de pacientes o si se les realizará una nefrectomía ya sea laparoscópica o mano asistida dependerá de la evaluación realizada previamente del paciente. Posteriormente esta indicación única iría ampliándose a pacientes con el riñón contralateral normal, esto no ocurre así en todos los casos.

La hidratación en el trasplante renal es muy importante. El nuevo riñón debe tener un riego adecuado para asegurar su funcionamiento óptimo. El volumen intravascular del paciente debe mantenerse por encima de lo normal para asegurar un buen flujo hacia el nuevo injerto renal. Un riñón trasplantado puede funcionar bien durante muchos años, dependiendo del estado de salud general del receptor y de las características del riñón trasplantado. A continuación, se detallará cada procedimiento para lograr manejar y entender de forma sencilla un tema interesante y complejo. 


\section{Procedimiento quirúrgico para pacientes sometidos a cirugía de trasplante renal}

Vol. 3, núm. 1., (2019)

José Fernando Calderón Ponce; César David Pibaque Tigua; Román Andrés Ortiz Burgasi; Miguel Ángel Sánchez Rodríguez; Luis Felipe Mendoza Alcívar; Maryury Beatriz Vinces Majojo

\section{Metodología}

Esta investigación está dirigida al estudio del "Procedimiento quirúrgico para pacientes sometidos a cirugía de trasplante renal". Para realizarlo se usó una metodología tipo descriptiva, con un enfoque documental, es decir, revisar fuentes disponibles en la red, como google académico, con contenido oportuno y relevante desde el punto de vista científico para dar respuesta a lo tratado en el presente artículo y que sirvan de inspiración para realizar otros proyectos. Las mismas pueden ser consultadas al final, en la bibliografía.

\section{Resultados.}

El trasplante renal consiste en la sustitución de los riñones nativos enfermos por un riñón sano, extraído de un donante vivo o muerto. La era moderna del trasplante renal comenzó a principios del siglo XX gracias al descubrimiento de nuevas técnicas de suturas, en particular los aportes de Ullman (1902) y Carrel (1914) permitieron establecer las bases de las anastomosis vasculares (Cubillos, 2018). En Colombia el primer trasplante renal exitoso de donante vivo se realizó el 29 de agosto de 1973 en Medellín por el grupo dirigido por los doctores Jaime Borrero y Álvaro Velásquez.

En la mayoría de las ciudades intermedias de Latinoamérica antes de 1990, las opciones terapéuticas para los pacientes con insuficiencia renal crónica eran limitadas, un pequeño porcentaje de pacientes recibía diálisis de forma regular porque existían pocas unidades renales en las principales ciudades del país, dificultando el desplazamiento de los pacientes, adicionalmente, se le adjudicaba tratamiento a jóvenes que tuvieran la insuficiencia renal como única patología, dejando como última opción el resto de la población.

En general todo paciente con insuficiencia renal terminal sin complicaciones o patologías graves asociadas que condicionen su supervivencia a corto plazo, con capacidad suficiente para entender y asumir el tratamiento, es candidato para recibir un trasplante renal. Como objetivos de esta valoración esta: la educación del paciente y su entorno familiar, detectar contraindicaciones 


\section{Procedimiento quirúrgico para pacientes sometidos a cirugía de trasplante \\ renal}

Vol. 3, núm. 1., (2019)

José Fernando Calderón Ponce; César David Pibaque Tigua; Román Andrés Ortiz Burgasi; Miguel Ángel Sánchez Rodríguez; Luis Felipe Mendoza Alcívar; Maryury Beatriz Vinces

Majojo

absolutas y relativas, evaluar los factores de riesgo, evaluar el estado psicológico y la capacidad de adhesión al tratamiento y resolver las alteraciones encontradas antes del trasplante

\section{Técnicas quirúrgicas.}

Trasplante renal clásico o heterotópico: es la técnica quirúrgica más utilizada y sencilla, consiste en la colocación del riñón a trasplantar en la fosa ilíaca derecha por vía extra peritoneal. Se realiza una incisión para rectal derecha externa en "palo de jockey" que por la parte superior no sobrepase el ombligo y por la inferior llegue hasta la sínfisis del pubis. Se incide la aponeurosis por la línea de Spiegel, se accede al espacio retroperitoneal, seccionando los vasos epigástricos y el ligamento redondo en la mujer, respetando el cordón espermático en el hombre. Se diseca la vena ilíaca externa, ligando los vasos linfáticos con seda. Se coloca el riñón sobre una almohadilla de granizado de hielo y se comienza la anastomosis venosa. Previo al inicio de la intervención el receptor recibe profilaxis antibiótica empírica de amplio espectro y se inicia la inmunosupresión; si el trasplante es de donante vivo ésta suele empezar una semana antes. (Truán, 2005).

Trasplante renal en posición lumbar baja: es la técnica que se anastomosa la vena renal a la vena cava o inicio de la vena iliaca primitiva y la arteria renal a la arteria ilíaca primitiva, pudiendo ser de utilidad en el caso de disponer de una vena renal muy corta, y teniendo el inconveniente de que se requiera trasplantar un uréter más largo. También la arteria ilíaca primitiva suele estar afecta con más frecuencia de ateromatosis en este lugar. Es una técnica actualmente poco utilizada, sustituida por la anastomosis a los vasos ilíacos externos.

Trasplante renal en fosa ilíaca izquierda u ortotópico: era práctica frecuente colocar los riñones derechos en la fosa ilíaca izquierda con la finalidad de disponer la vía urinaria siempre para que resultara más fácil las reintervenciones (resolución de fístulas, estenosis etc.). Después se ubicaba en la fosa ilíaca derecha, pero se invertían los riñones derechos con esa finalidad, dando lugar a una patología: la del riñón invertido, fundamentalmente ocasionado por dificultades de vaciado del injerto (Aguilera, Mayo 2009). Actualmente se colocan los riñones en fosa ilíaca 


\section{Procedimiento quirúrgico para pacientes sometidos a cirugía de trasplante renal}

Vol. 3, núm. 1., (2019)

José Fernando Calderón Ponce; César David Pibaque Tigua; Román Andrés Ortiz Burgasi; Miguel Ángel Sánchez Rodríguez; Luis Felipe Mendoza Alcívar; Maryury Beatriz Vinces Majojo

izquierda cuando está ocupada la derecha, o cuando existe una patología habitualmente vascular, que lo contraindique. Difiere fundamentalmente en que se necesita una vena renal más larga, y en caso de disponer de un riñón derecho, puede resultar dificultosa la anastomosis venosa.

Trasplante renal ortotópico izquierdo: describe una vía de abordaje extra peritoneal a los vasos esplénicos por Lumbotomía, utilizada inicialmente para la revascularización esplenorenal en la estenosis de la arteria renal, después para la derivación esplenorenal en la hipertensión portal y más tarde en el trasplante renal. El abordaje se realiza a través de una lumbotomía izquierda con resección de la décimo segunda costilla. Se rechaza el riñón hacia la línea media, se liga y secciona la arteria renal. Se diseca la vena renal cuidadosamente ligando todas sus colaterales hasta su bifurcación con la finalidad que sea lo más larga posible. La pelvis renal se diseca, seccionándola por sus infundíbulos, respetando posible tejido peripiélico.

Ubicada la arteria esplénica en la cola del páncreas, se libera ligando sus colaterales, hasta su bifurcación, donde se secciona previa ligadura de las mismas. La anastomosis venosa se realiza de forma terminal entre la vena renal del injerto y la vena renal del receptor, con dos suturas continuas de180 grados. La anastomosis arterial se realiza entre la arteria esplénica del receptor y la arteria renal del injerto de forma termino-terminal, previa espatulación de ambas bocas y con puntos sueltos para evitar estenosis posteriores. La vía urinaria se restablece mediante una anastomosis pielo piélica entre la pelvis del receptor y la pelvis del injerto, con dos suturas continuas de $180^{\circ}$, dejando autorizada la vía con un catéter doble $\mathrm{J}$ o una nefrostomía mínima.

Trasplante renal ortotópico izquierdo a vena esplénica: es una técnica apropiada para realizar un trasplante ortotópico. La vena renal no es viable porque existe una malformación, encontrándose varias venas pequeñas unidas entre sí, que no tienen una luz suficiente para permitir su anastomosis a la vena renal. En alguna ocasión puede darse un ovillo venoso de paredes muy finas por ejemplo en las agenesias de cava inferior, afortunadamente poco frecuente. En este caso, se puede anastomosar la vena renal a la vena esplénica tanto en término final como término lateral, sin necesidad de realizar una esplenectomía. 


\section{Procedimiento quirúrgico para pacientes sometidos a cirugía de trasplante \\ renal}

Vol. 3, núm. 1., (2019)

José Fernando Calderón Ponce; César David Pibaque Tigua; Román Andrés Ortiz Burgasi; Miguel Ángel Sánchez Rodríguez; Luis Felipe Mendoza Alcívar; Maryury Beatriz Vinces

Majojo

Una vez concluida la fase vascular se inicia la anastomosis de la vía urinaria, que ha de realizarse con especial cuidado puesto que las complicaciones urológicas son las más frecuentes de entre las complicaciones quirúrgicas. Puede hacerse mediante dos modalidades según se implante el uréter del injerto a la vejiga o a la vía propia del receptor, tanto a pelvis renal como a uréter.

\section{Ureteroneocistostomía}

La implantación uréterovesical mediante técnica antirreflujo es la forma más habitual de anastomosis urinaria y la que tiene menor índice de complicaciones existiendo dos posibilidades:

- Intra vesical en el que se hace una cistotomía y se labra un túnel submucoso de 2-3 cm, a través del cual se introduce el uréter, anastomosando su extremo al orificio de la mucosa, constituyéndose así el mecanismo anti reflujo, y cerrando después la cistotomía.

- Extra vesical, cada vez más utilizado, en el que se hace una miotomía del detrusor y posteriormente se abre un pequeño ojal en la mucosa vesical y se procede a la anastomosis con puntos sueltos del uréter a la mucosa vesical. Posteriormente se sutura el detrusor sobre la anastomosis, constituyendo el mecanismo antireflujo.

Todas estas maniobras se facilitan con el llenado previo de la vejiga con suero a través de la sonda. Todos los materiales de sutura empleados deben ser reabsorbibles para evitar la formación de litiasis. Debe tenerse cuidado de no rotar el uréter y dejar una longitud suficiente para que las suturas queden sin tensión.

Urétero uréterostomía y urétero pielostomía: se utiliza el uréter o pelvis renal de un riñón nativo del receptor. Sólo se emplea de primera intención cuando el uréter del injerto es excesivamente corto o tiene seriamente dañada su vascularización. Es preferible reservar esta técnica para reintervenciones tras complicaciones urinarias. La intervención finaliza con la colocación de un tubo de drenaje de ambiente, y cierre de capa muscular y fascia en dos planos de puntos sueltos de material reabsorbible. En el caso de donantes inferiores a un año de edad, los dos riñones del donante van a un mismo receptor mediante la anastomosis término- lateral de cava y aorta del 


\section{Procedimiento quirúrgico para pacientes sometidos a cirugía de trasplante renal}

Vol. 3, núm. 1., (2019)

José Fernando Calderón Ponce; César David Pibaque Tigua; Román Andrés Ortiz Burgasi; Miguel Ángel Sánchez Rodríguez; Luis Felipe Mendoza Alcívar; Maryury Beatriz Vinces Majojo

donante, con ligadura de sus extremos proximales, a los vasos iliacos del receptor, o bien la anastomosis por separado en serie sobre los vasos iliacos del receptor (García, Octubre 2003).

\section{Nefrectomía Laparoscópica}

La nefrectomía consiste en la extirpación quirúrgica de parte de un riñón (nefrectomía parcial), del riñón completo (nefrectomía simple) o del riñón, los ganglios linfáticos adyacentes y la glándula suprarrenal (nefrectomía radical). En una nefrectomía el cirujano corta los uréteres y los vasos sanguíneos y extrae el riñón. Puede hacerse con cirugía abierta o mediante laparoscopia. La cirugía abierta se realiza a través de una incisión en el costado o en la parte frontal del abdomen, mientras que la cirugía laparoscópica solo requiere de tres o cuatro pequeños cortes en el abdomen y en el costado, por lo que la recuperación es mucho más rápida (Abascal, 2012). La nefrectomía se realiza bajo anestesia general y suele durar en torno a tres horas. Tras la intervención, los pacientes deben permanecer ingresados entre 2 y 7 días en función de la técnica empleada.

El abordaje laparoscópico en una nefrectomía radical puede ser transperitoneal o accediendo directamente al retro peritoneo (lumboscopia). El más aplicado de los dos es el transperitoneal debido a que proporciona mayor espacio de trabajo y mejor orientación en el campo quirúrgico. La lumboscopia permite trabajar directamente en el retro peritoneo; la cavidad peritoneal permanece intacta, lo que se traduce en una menor distensión abdominal en el postoperatorio, menor riesgo de lesión intestinal y de órganos intra abdominales. Una tercera posibilidad es el acceso transperitoneal asistido por la mano. La principal ventaja es que la mano sirve de referencia en la pérdida de la tercera dimensión y la mayor seguridad que proporciona el hecho de tener la mano en el campo operatorio, en caso de tener un accidente vascular, sin duda es la principal preocupación en la cirugía retroperitoneal. Como desventaja, la disección es peor, debido a que se tiene una mano ocupada permanentemente, sin poder emplearla para la disección más fina (Aguilera, Mayo 2009).

\section{Vía transperitoneal}




\section{Procedimiento quirúrgico para pacientes sometidos a cirugía de trasplante \\ renal}

Vol. 3, núm. 1., (2019)

José Fernando Calderón Ponce; César David Pibaque Tigua; Román Andrés Ortiz Burgasi; Miguel Ángel Sánchez Rodríguez; Luis Felipe Mendoza Alcívar; Maryury Beatriz Vinces

Majojo

Se exponen los distintos pasos que realiza para la misma un grupo de médicos, se reconoce que no es una metodología única a los mismos podrían añadirse muchas variantes válidas (Contreras, 2011):

- Colocación del paciente y cirujanos lumbotomía clásica: el cirujano se coloca de pie frente al abdomen del paciente, su asistente es preferible que esté sentado y la instrumentista debe tener accesibilidad a manejar el cuarto trócar (vigilar la fijación y almohadillado del paciente).

- Creación del pneumoperitoneo y colocación de trócares; se usan cuatro trócares. El primero de $10 \mathrm{~mm}$ se sitúa en condiciones estándar para umbilical en la línea externa del recto del abdomen y por él se colocará la óptica. Esta puede ser de 0 o 30 grados, en la fosa ilíaca y en hipocondrio ipsilaterales se colocan el segundo y tercer trócares, asignando a la mano dominante del cirujano el trócar de $12 \mathrm{~mm}$ que permita el uso de endogias y a la otra uno de $5 \mathrm{~mm}$. El cuarto trócar, de $5 \mathrm{~mm}$, se coloca en la línea axilar anterior y lo usa el ayudante/instrumentista.

- Decolación colon derecho/izquierdo.

- Creación plana entre cara anterior renal y colon; maniobra de Köcher en el lado derecho, desplazamiento del paquete es pleno pancreático en el izquierdo.

- Individualización de vena gonadal y uréter; a excepción de variantes, la gonadal siempre se encontrará primero y en una situación más externa a la línea media en lumbotomía. Es clave para localizar la vena renal izquierda o la cava.

- Acceso ascendente hacia el hilio renal; mediante esta maniobra se pueden encontrar pedículos polares inferiores que deben ser correctamente controlados. En laparoscopia es frecuente observar la arteria gonadal, que puede controlarse con bipolar o clips. 


\section{Procedimiento quirúrgico para pacientes sometidos a cirugía de trasplante renal}

Vol. 3, núm. 1., (2019)

José Fernando Calderón Ponce; César David Pibaque Tigua; Román Andrés Ortiz Burgasi; Miguel Ángel Sánchez Rodríguez; Luis Felipe Mendoza Alcívar; Maryury Beatriz Vinces Majojo

- Identificación, control y sección de arteria y vena; la disección de los elementos del hilio debe hacerse siguiendo los principios de cirugía abierta.

- Sección de uréter.

- Liberación del polo superior; siguiendo los criterios oncológicos establecidos, la glándula suprarrenal se incluye o no en la pieza. La maniobra se ve facilitada con la óptica de 30 grados y en el lado izquierdo con la movilización previa del eje espleno pancreático.

- Exéresis de la pieza embolsada.

\section{Vía retroperitoneal}

La generación del neumoperitoneo puede realizarse bajo visión directa con una pequeña incisión de 1-2 cm o a ciegas con la aguja de Veress. Permite un acceso más rápido, pero al ser una técnica cerrada requiere ser aplicada por cirujanos con experiencia. En caso de pacientes con antecedentes quirúrgicos abdominales, exige vigilar el riesgo de lesión intestinal por adherencias (National Kidney Foundation, 2019).

Creado el neumoperitoneo y colocados los puertos de trabajo, es importante la orientación anatómica. La identificación del músculo psoas, del uréter y vena gonadal parece la vía más segura para desarrollar el plano que nos ubicará al hilio renal, variando si se corresponde al lado derecho o izquierdo, peculiaridades anatómicas a considerar; presencia de venas lumbares, proximidad de los grandes vasos, venas suprarrenales, etc.

Retirada la pieza, es conveniente revisar la hemostasia del lecho quirúrgico, a fin de reducir la presión intra abdominal durante unos minutos, que permitirá visualizar el sangrado venoso que podría estar colapsado por la presión. También se debe revisar la retirada de los trócares para evitar producir sangrado en este punto. La extracción de la pieza se hace con bolsa. 


\section{Procedimiento quirúrgico para pacientes sometidos a cirugía de trasplante \\ renal}

Vol. 3, núm. 1., (2019)

José Fernando Calderón Ponce; César David Pibaque Tigua; Román Andrés Ortiz Burgasi; Miguel Ángel Sánchez Rodríguez; Luis Felipe Mendoza Alcívar; Maryury Beatriz Vinces

Majojo

Se evidencia que en la vía retroperitoneal el control hiliar y el tiempo quirúrgico es ligeramente más rápido, recomendándola para los pacientes obesos y para aquellos con cirugías abdominales previas. La vía transperitoneal, por el contrario, se recomienda para aquellos pacientes que han sufrido cirugía retroperitoneal, para tumores grandes o nefrectomía radical laparoscópica cito reductoras en pacientes metastásicos, para nefrectomías parciales, de donante vivo y cuando sea posible utilizar robótica o dispositivos mano asistida. En los pacientes con tumores renales se aconseja llevarse un parche anterior de peritoneo por la posibilidad de márgenes positivos a dicho nivel (Briones, Mayo 2006).

Ablación donante cadavérico: se realiza en pacientes con muerte encefálica y aun con corazón batiente, serán considerados donantes de órganos todos aquellos cadáveres en situación de muerte encefálica por traumatismo craneoencefálico, accidente cerebrovascular, anoxia cerebral o tumores cerebrales no metastáticos.

- Disección ángulo hepático colon hasta el ciego.

- Ligadura y sección vena mesentérica inferior.

- Maniobra de Kocher en el duodeno.

- Exposición vena cava y vena. renal.

- Ligadura y sección arteria mesentérica superior.

- Reparos vena cava, arteria aorta.

- Ligaduras vena gonadal.

- Ligaduras proximales fuertes de venas. y arterias.

- Ligadura arteria distal, sección, y canulación.

- Ligaduras de los reparos.

- Canulación, perfusión cardiopléjica.

- Inicio de perfusión demás órganos.

- Decolamiento izquierdo.

- Sección de grandes vasos y disección en bloque por posterior.

- Sección vena y arteria renal. 


\section{Procedimiento quirúrgico para pacientes sometidos a cirugía de trasplante renal}

Vol. 3, núm. 1., (2019)

José Fernando Calderón Ponce; César David Pibaque Tigua; Román Andrés Ortiz Burgasi; Miguel Ángel Sánchez Rodríguez; Luis Felipe Mendoza Alcívar; Maryury Beatriz Vinces Majojo

\section{Complicaciones.}

Complicaciones vasculares (Castillo, Agosto 2008):

- Hemorragia incoercible, tanto durante el acto quirúrgico como en el postoperatorio (ya sea por ruptura renal o de sus vasos).

- Trombosis de los vasos renales, tanto de la arteria como de la vena, que en la mayoría de los casos obligan a la extirpación del riñón.

- Estenosis de la arteria renal (estrechez) y estenosis y/o trombosis de la arteria ilíaca con efectos secundarios sobre miembros inferiores como claudicación e isquemia.

Complicaciones urológicas:

- Hematuria con necesidad de realizar lavados frecuentes por sonda vesical.

- Fístulas urinarias a cualquier nivel del tracto urinario, que en la mayoría de las ocasiones obligan a realizar reintervenciones.

- Estenosis u obstrucción a cualquier nivel del tracto urinario, que obligue a intervenciones quirúrgicas u otros procedimientos.

- Retención urinaria.

Complicaciones como consecuencia de la apertura del abdomen y de la cirugía del trasplante.

- Íleo paralitico pasajero o persistente.

- Obstrucción o perforación intestinal que precise la práctica de re intervención.

- Peritonitis.

- Infección de la herida quirúrgica.

- Dehiscencia de sutura que puede necesitar una intervención secundaria, evisceración intestinal, eventración, fístulas intestinales permanentes o temporales, defectos estéticos derivados de alguna de las complicaciones anteriores o procesos cicatrización anormal.

- Lesiones vasculares severas (arterias aorta, ilíacas, hipogástricas; venas cavas e ilíacas).

- Lesiones ureterales (fundamentalmente obstrucción urinaria). 


\section{Procedimiento quirúrgico para pacientes sometidos a cirugía de trasplante \\ renal}

Vol. 3, núm. 1., (2019)

José Fernando Calderón Ponce; César David Pibaque Tigua; Román Andrés Ortiz Burgasi; Miguel Ángel Sánchez Rodríguez; Luis Felipe Mendoza Alcívar; Maryury Beatriz Vinces

Majojo

- Lesión nerviosa.

- Fibrosis reactiva y estenosis de uréter y de los vasos tanto arteriales como venosos, a largo plazo.

- Problemas derivados de la linfadenectomía.

- Linfocele (colección de linfa), que puede producir obstrucción urinaria y venosa (que puede facilitar la aparición de trombosis en las mismas), que requiere frecuentemente la realización de manipulaciones (punción-drenaje) posteriores e incluso nueva re intervención quirúrgica (derivación a peritoneo).

- Linfedema de la extremidad inferior ipsilateral.

Cualesquiera de las complicaciones anteriores pueden poner en peligro la funcionalidad y viabilidad del riñón, haciendo necesarias otras intervenciones para su solución, pudiendo incluso ser necesario, extirpar el riñón o tener consecuencias muy diversas, oscilando desde una gravedad mediana hasta la posibilidad real de muerte, como consecuencia directa de la complicación o por efectos secundarios de tratamientos empleados.

\section{Conclusión.}

El trasplante renal consiste en la sustitución de los riñones nativos enfermos por un riñón sano, extraído de un donante vivo o muerto. Todo paciente con insuficiencia renal terminal sin complicaciones o patologías graves asociadas que condicionen su supervivencia a corto plazo, con capacidad suficiente para entender y asumir el tratamiento, es candidato para recibir un trasplante renal. Como objetivos de esta valoración esta: la educación del paciente y su entorno familiar, detectar contraindicaciones absolutas y relativas, evaluar los factores de riesgo, evaluar el estado psicológico y la capacidad de adhesión al tratamiento y resolver las alteraciones encontradas antes del trasplante.

La heterotópico es la técnica quirúrgica más utilizada y sencilla, consiste en la colocación del riñón a trasplantar en la fosa ilíaca derecha por vía extra peritoneal. La_ortotópico: consiste en colocar los riñones derechos en la fosa ilíaca izquierda con la finalidad de disponer la vía urinaria 


\section{Procedimiento quirúrgico para pacientes sometidos a cirugía de trasplante renal}

Vol. 3, núm. 1., (2019)

José Fernando Calderón Ponce; César David Pibaque Tigua; Román Andrés Ortiz Burgasi; Miguel Ángel Sánchez Rodríguez; Luis Felipe Mendoza Alcívar; Maryury Beatriz Vinces Majojo

siempre para que resultara más fácil las reintervenciones (resolución de fístulas, estenosis etc.). El trasplante renal ortotópico izquierdo describe una vía de abordaje extra peritoneal a los vasos esplénicos por Lumbotomía, utilizada inicialmente para la revascularización esplenorenal en la estenosis de la arteria renal, después para la derivación esplenorenal en la hipertensión portal y más tarde en el trasplante renal.

La nefrectomía consiste en la extirpación quirúrgica de parte de un riñón (nefrectomía parcial), del riñón completo (nefrectomía simple) o del riñón, los ganglios linfáticos adyacentes y la glándula suprarrenal (nefrectomía radical). En una nefrectomía el cirujano corta los uréteres y los vasos sanguíneos y extrae el riñón. Puede hacerse con cirugía abierta o mediante laparoscopia. La cirugía abierta se realiza a través de una incisión en el costado o en la parte frontal del abdomen, mientras que la cirugía laparoscópica solo requiere de tres o cuatro pequeños cortes en el abdomen y en el costado, por lo que la recuperación es mucho más rápida. El abordaje laparoscópico en una nefrectomía radical puede ser transperitoneal o accediendo directamente al retro peritoneo (lumboscopia).

Algunas complicaciones del procedimiento quirúrgico son: hemorragia incoercible, tanto durante el acto quirúrgico como en el postoperatorio (ya sea por ruptura renal o de sus vasos), trombosis de los vasos renales, tanto de la arteria como de la vena, que en la mayoría de los casos obligan a la extirpación del riñón, fístulas urinarias a cualquier nivel del tracto urinario, peritonitis, lesiones vasculares severas (arterias aorta, ilíacas, hipogástricas; venas cavas e ilíacas). Cualquiera de las complicaciones anteriores puede poner en peligro la funcionalidad y viabilidad del riñón, haciendo necesarias otras intervenciones para su solución, pudiendo incluso ser necesario, extirpar el riñón o tener consecuencias muy diversas, oscilando desde una gravedad mediana hasta la posibilidad real de muerte.

\section{Bibliografía.}

Abascal, J. (Mayo de 2012). TRANSPLANTE RENAL. Obtenido de Fundación Renal Iñigo Alvarez de Toledo: www.fundacionrenal.com 


\section{Procedimiento quirúrgico para pacientes sometidos a cirugía de trasplante}

renal

Vol. 3, núm. 1., (2019)

José Fernando Calderón Ponce; César David Pibaque Tigua; Román Andrés Ortiz Burgasi; Miguel Ángel Sánchez Rodríguez; Luis Felipe Mendoza Alcívar; Maryury Beatriz Vinces

Majojo

Aguilera, A. (Mayo 2009). Nefrectomía radical laparoscopica. Técnica, resultados y complicaciones. Actas de Urología Española Volumen 33 No.5, www.scielo.isciii.es.

Briones, R. (Mayo 2006). Nefrectomía radical laparoscópica. Actas de Urología Española Volumen $30 N^{\circ}(5), 479-491$ www.scielo.isciii.es.

Castillo, O. (Agosto 2008). Nefrectomía radical laparoscópica. Revista Chilena de Cirugía Volumen $60 N^{\circ} 4$, 297-303 www.scielo.conicyt.cl.

Contreras, J. (23 de Marzo de 2011). Complicación durante una Nefrectomía Laparoscópica. Obtenido de AnestesiaR: www.anestesiar.com

Cubillos, J. (2018). ASPECTOS QUIRURGICOS DEL TRASPLANTE RENAL. Nefrología Básica $N^{\circ}$ 2, 435-443 www.asocolnef.com.

García, A. (Octubre 2003). TRANSPLANTE RENAL. TÉCNICA Y COMPLICACIONES. Actas Urología Españolas Vol.27 No.9, www.scielo.isciii.es.

National Kidney Foundation. (Enero de 2019). Transplante de Riñón. Obtenido de National Kidney Foundation: www.kidney.org

Truán, D. (2005). TRASPLANTE RENAL DE DONANTE VIVO: IMPLANTE RENAL Y SUS VARIANTES . Archivo Español Urología Volumen 58, $N^{\circ}$ 6, 521-530 www.scielo.isciii.es.

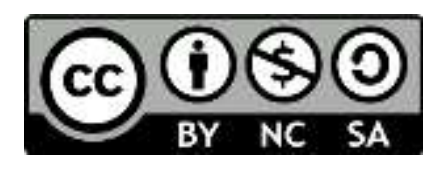

RECONOCIMIENTO-NOCOMERCIAL-COMPARTIRIGUAL

CC BY-NC-SA

ESTA LICENCIA PERMITE A OTROS ENTREMEZCLAR, AJUSTAR Y CONSTRUIR A PARTIR DE SU OBRA CON FINES NO COMERCIALES, SIEMPRE Y CUANDO LE RECONOZCAN LA AUTORÍA Y SUS NUEVAS CREACIONES ESTÉN BAJO UNA LICENCIA CON LOS MISMOS TÉRMINOS. 